\title{
Numerical analysis of flow characteristics of Jeffery nanofluid past a moving plate in conducting field
}

\author{
P.S.Rao ${ }^{1+}$, B. Hari Babu ${ }^{2}$ and S.V.K. Varma ${ }^{3}$ \\ ${ }^{1}$ Department of Applied Mathematics, Indian Institute of Technology (I.S.M), Dhanbad-826004, Jharkand., India. \\ ${ }^{2}$ Research Scholar, Indian Institute of Technology (I.S.M), Dhanbad-826004, Jharkand., India. \\ ${ }^{3}$ Department of Mathematics, S.V. University, Tirupati-517502, A.P., India.
}

†Corresponding Author Email: psrao@iitism.ac.in

\begin{abstract}
This paper reveals the physical properties of Jeffery nanofluid flow past a moving plate embedded in porous medium under the existence of radiation and thermal diffusion. The analysis is carried out in three cases of moving plate, namely stationary plate $(\lambda=0)$, forth-moving plate $(\lambda=1)$, back-moving plate $(\lambda=-1)$. Finite difference method is applied to solve the governing equations of the flow and pointed out the variations in velocity, temperature and concentration with the use of graphical presentations. The impact of several parameters on local skin friction, Nusselt number and Sherwood number is also noticed and discussed. Enhancement of velocity is observed under the impact of Jeffery parameter for the cases of stationary plate and back-moving plate, whereas reverse nature is found in the case of forth-moving plate. The velocity enhances as the values of porosity parameter increases for the case of stationary plate and forth-moving plate but a reverse nature is noticed in the case of back-moving plate.
\end{abstract}

Keywords: Jeffery nanofluid, radiation, thermal diffusion, finite difference method, moving plate and porous medium.

\section{NOMENCLATURE}

$\begin{array}{ll}u^{*} & \text { velocity component along the x-direction }\left[\mathrm{m} \cdot \mathrm{s}^{-1}\right] \\ T^{*} & \text { temperature }[\mathrm{K}] \\ C^{*} & \text { concentration }[\mathrm{g} / \mathrm{ml}] \\ \mu_{n f} & \text { dynamic viscosity of the nanofluid [Pa.s }] \\ \rho_{n f} & \text { density of the nanofluid }\left[\mathrm{kg} \cdot \mathrm{m}^{-3}\right] \\ \sigma_{n f} & \text { electrical conductivity of the nanofluid }\left[\mathrm{ohm}^{-1} \mathrm{~s}^{-1}\right] \\ k_{n f} & \text { thermal conductivity of the nanofluid }\left[\mathrm{W} \cdot \mathrm{m}^{-1} \cdot \mathrm{K}^{-1}\right] \\ g & \text { acceleration due to gravity }\left[\mathrm{m} \cdot \mathrm{s}^{-2}\right] \\ q_{r} & \text { radiative heat flux } \\ \left(\rho C_{p}\right)_{n f} & \text { heat capacitance of the nanofluid } \\ \beta_{n f} & \text { thermal expansion coefficient }\left[\mathrm{K}^{-1}\right] \\ \beta_{n f}^{*} & \text { mass transfer coefficient }[\mathrm{m} / \mathrm{s}] \\ \phi & \text { solid volume fraction of the nanoparticles }[\mathrm{m} . \mathrm{g}] \\ M & \text { magnetic parameter } \\ R & \text { Radiation parameter } \\ \mathrm{Pr} & \text { Prandtl number } \\ K & \text { porosity parameter } \\ G r & \text { Grashof number } \\ G c & \text { modified Grashof number } \\ & \end{array}$

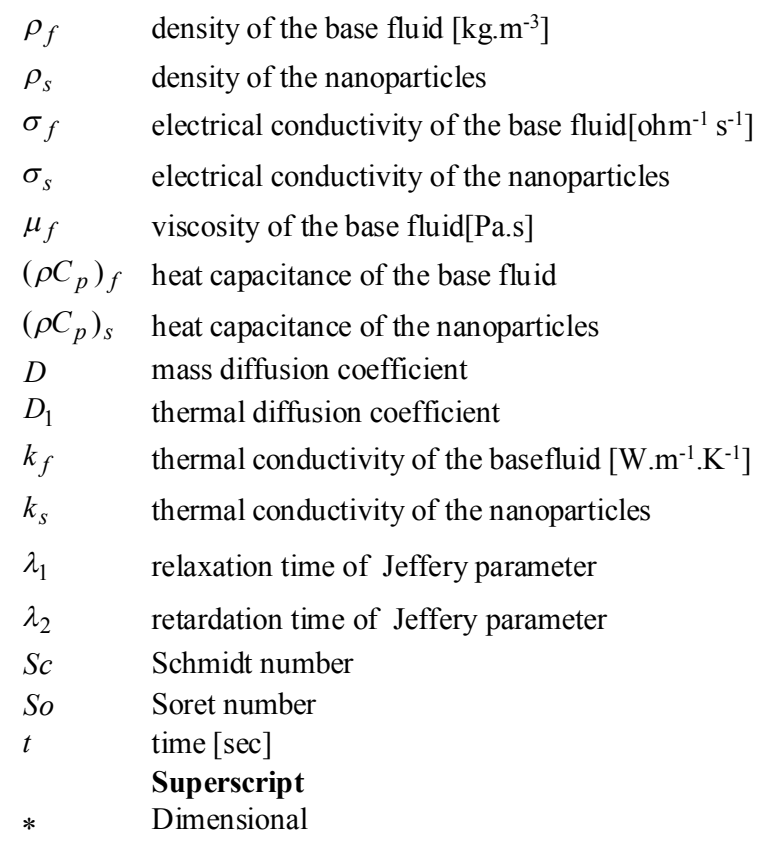




\section{INTRODUCTION}

An effectual fluid is concerned in various engineering and industrial purposes that are either in flowing or stagnant state. This functioning fluid is used to transfer energy from one position to the other. The tolerable heat transmission performance has been a most important issue for a long period and it can be determined by means of a new effective fluid that has superior thermal performance compared to ordinary base liquids. Recently nanofluid came into existence to take place of working fluid. Nanofluid is a fluid formed by submerging the nanoparticles of size $1-100 \mathrm{~nm}$ into a base fluid. The thermal conductivity of these fluids is higher than that of base fluids like water, kerosene etc. Further, the novel properties of Brownian motion and thermophoresis of such fluids make them potentially useful. Nanoparticles are used to enhance the thermal characteristics of ordinary base fluids such as water, ethylene glycol or oil. In addition the magneto-nanofluid is a unique material that has both liquid and magnetic properties. Such nanofluid has superficial role in construction of loud speakers, blood analysis and cancer therapy. Choi (1995) stated the details of enhancing thermal conductivity of fluids with nanoparticles developments and applications of non-Newtonian flows. Eastman et al. (2001) established anomalously enhanced effective thermal conductivities of ethylene glycol-based nanofluids containing copper nanoparticles. Further Choi et al. (2001) reported anomalously thermal conductivity enrichment in nanotube suspensions. Das and Choi (2009), Das and Jana (2015), Kakac and Pramuanjaroenkij (2009) examined and discussed natural convective magnetonanofluid flow and radiative heat transfer past a moving vertical plate. Sheikholeslami et al. $(2016,2017)$ reported on external magnetic field impact on water based nanofluid and transportation of MHD nanofluid over a porous partly annulus with numerical approach. Syakila and Pop (2010) examined and revealed mixed convection boundary layer flow from a vertical flat plate implanted in a porous medium filled with nanofluids.

Many researchers showed interest on studying the non-Newtonian fluids because of their significance in manufacturing, industrial and technological applications. Such fluids have a nonlinear relationship between the stress and the rate of strain. Jeffrey model is considered as the simplest non-Newtonian fluid model. It is chosen to illustrate the flows of physiological fluids in tubes as well as channels. Jeffrey model considers a linear model in which the time derivatives are applied instead of convected derivatives. The notable industrial applications of these fluids are polymer systems (melt and solutions) and multiphase system such as foams, emulsions, and slurries. Hayat et al. (2012) established and derived the properties of a
Jeffrey fluid with convective boundary conditions. Maqbool et al. (2017) considered and analyzed these flow embedded in a porous medium with ramped wall velocity and temperature. Sreenadh et al. (2015) deliberated the nature of MHD free surface flow of a Jeffery fluid over a deformable porous layer. Al-Khafajy et al. (2016) selected and evaluated the MHD oscillatory flow of this fluid with variable viscosity. Kavita et al. (2012) reported the same analysis over a channel. Mabood et al. (2016) discussed numerical study of these fluids with magnetic field effect and variable fluid properties.

Further researchers paid interest on studying Jeffery nanofluids by considering various parameters and boundaries. Shehzad et al. $(2014,2015,2016)$ investigated and reported MHD flow of Jeffrey nanofluid by implementing convective boundary conditions, nonlinear thermal radiation in three-dimensional flow with internal heat generation and magnetic field. Abbasi et al. (2015) considered and examined the flow of Jeffrey nanofluid by using heat and mass flux conditions. Dalir et al. (2015) reported theoretically the entropy analysis of this flow over a stretching sheet. Hayat et al. $(2015,2016)$ discussed mathematically on three-dimensional boundary layer flow of Jeffrey nanofluid and also studied with a new mass flux condition. Khan (2015) established a note on exact solutions for the unsteady free convection flow of a Jeffrey fluid. Mehmood et al. (2017) scrutinized flow and heat transfer analysis of Jeffery nano fluid impinging obliquely over a stretched plate. Feroz Ahmed Soomro et al. (2017a, 2017b) evaluated numerical study of entropy generation in MHD water based carbon nanotubes along an inclined permeable surface and also studied passive control of nanoparticles due to convective heat transfer of Prandtl fluid model at the stretching surface. Ganesh Kumar et al. (2017) reported effects of the same flow over a flat plate in the presence of chemical reaction. Hashim et al. (2017) deliberated the dual solutions in flow of a non-Newtonian fluid with homogeneous heterogeneous reactions: Critical points. Ramzan et al. (2017) examined effects of thermal and solutal stratification on Jeffrey magneto-nanofluid along an inclined stretching cylinder with thermal radiation and heat generation/absorption. Rashidi et al. (2017) investigated MHD flow and heat transfer characteristics of Williamson nanofluid over a stretching sheet with variable thickness and variable thermal conductivity. Rohit Sharma et al. (2017) considered and analyzed radiative magnetonanofluid over an accelerated plate in a rotating medium with Hall effects. Seth and Mishra (2017) studied analysis of transient flow of MHD nanofluid past a nonlinear stretching sheet considering Navier's slip boundary condition. Seth et al. (2016, 2017a, 2017b) established the studies on MHD convective flows with various considerations like rotation, ramped wall temperature, Hall 
effect and accelerated plate. Chandra Reddy et al. (2015) explained thermal and solutal buoyancy effect on heat cause visco-elastic MHD boundary layer flow under varying suction. Singh et al. (2017) analyzed unsteady MHD natural convective flow of a rotating fluid over an infinite vertical plate due to oscillatory movement of the free-stream with hall and ion-slip currents.

In view of the literature survey, we made an extension to the report of Das and Jana (2015) which describes MHD nanofluid flow past a moving plate. The novelty of this work is the implementation of mass transfer effects on Jeffery parameter with radiation and thermal diffusion embedded in porous medium. The Copper-water nanofluid with Jeffery parameter is considered and analyzed theoretically.

\section{PROBLEM FORMULATION}

We have considered Jeffery nanofluid flow past a moving plate embedded in porous medium under the existence of radiation and thermal diffusion. The analysis is carried out in three cases of moving plate, namely stationary plate $(\lambda=0)$, forth-moving plate $(\lambda=1)$, backmoving plate $(\lambda=-1)$. At time $\mathrm{t}^{*}=0$, the plate is assumed to be at rest with the constant ambient temperature $T_{\infty}^{*}$ and the concentration $C_{\infty}^{*}$. At time $\mathrm{t}^{*}>0$, the plate starts moving in its own plane with the velocity $\lambda \mathrm{u}_{0}$ in the vertical direction, where $\mathrm{u}_{0}$ is constant and the temperature of the plate is raised or lowered to $T_{w}^{*}$ and the level of concentration is also maintained at $C_{w}^{*}$. We choose the $\mathrm{x}$ axis along the plate in the upward direction vertically and $\mathrm{y}$-axis is taken perpendicular to it. A uniform transverse magnetic field of strength $\mathrm{B}_{0}$ is applied perpendicular to the plate (parallel to the y-axis). The plate coincides with the plane $y=0$ and the fluid flow being restricted to $y>0$. It is assumed that the pressure gradient is neglected in this analysis. It is also assumed that a radiative heat flux $\mathrm{q}_{\mathrm{r}}$ is applied in the normal direction to the plate and mass flux caused by the temperature differences which is termed as thermal diffusion effect is considered. The fluid is water based Jeffery nanofluid containing nanoparticles of Copper $(\mathrm{Cu})$. It is further assumed that the base fluid and the suspended nanoparticles are in thermal equilibrium. The values related to thermo physical properties of the cu-water nanofluid are given in Table 1. We assume that the density is linearly dependent on temperature buoyancy forces in the equations of flow pattern. This consideration is exact enough for both dropping liquid and gases for minor values of the temperature difference.

It is assumed that induced magnetic field generated by the fluid flow is negligible in comparison with the applied one so that we assume magnetic field $\vec{B} \equiv\left(0,0, B_{0}\right)$. This assumption is relevant since the magnetic Reynolds number is very less for metallic liquids and partially ionized fluids.

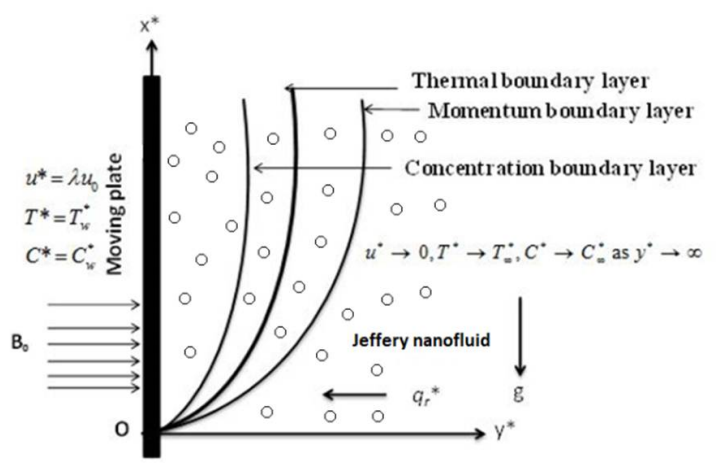

Fig. 1. Physical model and coordinate system

Further no external electric field is applied such that the effect of polarization of fluid is negligible (Das and Jana (2015)) and hence we assume $\vec{E}=(0,0,0)$.

The modified Darcy's law for the Jeffery fluid is given by Mehmood et al. (2017).

$$
r=\frac{-\mu \phi}{k\left(1+\lambda_{1}\right)}\left(1+\lambda_{2} \frac{\partial}{\partial t}\right) V
$$

Where $\lambda_{1}$ and $\lambda_{2}$ are the relaxation and retardation times.

Under the assumption of a small magnetic Reynolds number and Boussinesq's approximation the governing equations are [Das and Jana (2015) and Mehmood et al. (2017)].

$$
\begin{gathered}
\rho_{n f} \frac{\partial u^{*}}{\partial t^{*}}=\frac{\mu_{n f}}{1+\lambda_{1}}\left(1+\lambda_{2} \frac{\partial}{\partial t^{*}}\right) \frac{\partial^{2} u^{*}}{\partial y^{*^{2}}}-\sigma_{n f} B_{0}^{2} u^{*} \\
+g(\rho \beta)_{n f}\left(T^{*}-T_{\infty}^{*}\right)-\frac{\mu_{n f}}{k_{p}^{*}} u^{*} \\
+g\left(\rho \beta^{*}\right)_{n f}\left(C^{*}-C_{\infty}^{*}\right) \\
\left(\rho C_{p}\right)_{n f} \frac{\partial T^{*}}{\partial t^{*}}=k_{n f} \frac{\partial^{2} T^{*}}{\partial y^{*^{2}}}-\frac{\partial q_{r}^{*}}{\partial y} \\
\frac{\partial C^{*}}{\partial t^{*}}=D \frac{\partial^{2} C^{*}}{\partial y^{*^{2}}}+D_{1} \frac{\partial^{2} T^{*}}{\partial y^{*^{2}}}
\end{gathered}
$$

where $\mathrm{u}^{*}$ is the velocity components along the $\mathrm{x}$-direction,

$$
\begin{aligned}
& \mu_{n f}=\frac{\mu_{f}}{(1-\phi)^{2.5}}, \quad \rho_{n f}=(1-\phi) \rho_{f}+\phi \rho_{s}, \\
& \left(\rho C_{p}\right)_{n f}=(1-\phi)\left(\rho C_{p}\right)_{f}+\phi\left(\rho C_{p}\right)_{s}, \\
& (\rho \beta)_{n f}=(1-\phi)(\rho \beta)_{f}+\phi(\rho \beta)_{s} \\
& \left(\rho \beta^{*}\right)_{n f}=(1-\phi)\left(\rho \beta^{*}\right)_{f}+\phi\left(\rho \beta^{*}\right)_{s}, \\
& \sigma_{n f}=\sigma_{f}\left[1+\frac{3(\sigma-1) \phi}{(\sigma+2)-(\sigma-1) \phi}\right], \quad \sigma=\frac{\sigma_{s}}{\sigma_{f}},
\end{aligned}
$$

The effective thermal conductivity of the nanofluid given by Hamilton and Crosser model followed by Kakac and Pramuanjaroenkij (2009) is given by 


$$
k_{n f}=k_{f}\left[\frac{k_{s}+2 k_{f}-2 \phi\left(k_{f}-k_{s}\right)}{k_{s}+2 k_{f}+\phi\left(k_{f}-k_{s}\right)}\right]
$$

where the subscripts $n f, f$ and $s$ denote the thermo physical properties of the nanofluid, base fluid and nanoparticles, respectively.

The initial and boundary conditions are

$$
\begin{aligned}
& t^{*}=0: u^{*}=0, T^{*}=T_{\infty}^{*}, C^{*}=C_{\infty}^{*} \text { for all } y^{*} \geq 0, \\
& t^{*}>0: u^{*}=\lambda u_{0}, T^{*}=T_{w}^{*}, C^{*}=C_{w}^{*} \text { at } y^{*}=0, \\
& t^{*}>0: u^{*} \rightarrow 0, T^{*} \rightarrow T_{\infty}^{*}, C^{*} \rightarrow C_{\infty}^{*} \text { as } y^{*} \rightarrow \infty
\end{aligned}
$$

where $\lambda$ indicates the direction of the moving plate with $\lambda=0$ for the stationary plate, while $\lambda= \pm 1$ for the forth and back movement of the plate.

Table 1: Thermo physical properties of water and nanoparticle Das and Jana (2015).

\begin{tabular}{|c|c|c|}
\hline $\begin{array}{c}\text { Physical } \\
\text { properties }\end{array}$ & $\begin{array}{c}\text { Water/base } \\
\text { fluid }\end{array}$ & Cu(copper) \\
\hline$\rho\left(\mathrm{kg} / \mathrm{m}^{3}\right)$ & 997.1 & 8933 \\
\hline$C_{p}(\mathrm{~J} / \mathrm{kg} \mathrm{K})$ & 4179 & 385 \\
\hline $\mathrm{k}(\mathrm{W} / \mathrm{m} \mathrm{K})$ & 0.613 & 401 \\
\hline$\beta \times 10^{5}\left(K^{-1}\right)$ & 21 & 1.67 \\
\hline$\phi$ & 0.0 & 0.05 \\
\hline$\sigma(\mathrm{S} / \mathrm{m})$ & $5.5 \times 10^{-6}$ & $59.6 \times 10^{6}$ \\
\hline
\end{tabular}

It is assumed that the fluid is an optically thick (photon mean free path is very small) gray gas (which emits and absorbs but does not scatter thermal radiation). In an optically thick medium the radiation penetration length is small compare to the characteristic length. The photon mean path is the average distance travelled by a moving photon between successive collisions which modify its direction or energy or other particle properties. For an optically thick fluid, we can adopt Rosseland approximation for radiative flux. The Rosseland approximation from Das and Jana [5] applies to optically thick media and gives the net radiation heat flux $q_{r}$ by the expression

$q_{r}^{*}=-\frac{4 \sigma^{*}}{3 k^{*}} \frac{\partial T^{4}}{\partial y}$

Where $\sigma^{*}\left(=5.67 \times 10^{-8} \mathrm{~W} / \mathrm{m}^{2} \mathrm{~K}^{4}\right)$ is the StefanBoltzmann constant and $k^{*}\left(\mathrm{~m}^{-1}\right)$ the Rosseland mean obsorption coefficient. We assume that the temperature difference within the flow is sufficiently small such that the term $T^{4}$ may be expressed as a linear function of temperature. This is done by expanding $T^{4}$ in a Taylor series about a free stream temperature $T_{\infty}$ as follows: $T^{4}=T_{\infty}^{4}+3 T_{\infty}^{3}\left(T-T_{\infty}\right)+6 T_{\infty}^{2}\left(T-T_{\infty}\right)^{2}+$

Neglecting higher-order terms in equation (8) beyond the first order in $\left(T-T_{\infty}\right)$, we get

$$
T^{4} \approx 4 T_{\infty}^{3} T-3 T_{\infty}^{4}
$$

Using the Eq.s (7) and (9), Eq. (2) becomes

$$
\frac{\partial T^{*}}{\partial t^{*}}=\frac{1}{\left(\rho C_{p}\right)_{n f}}\left(k_{n f}+\frac{16 \sigma^{*} T_{\infty}^{3}}{3 k^{*}}\right) \frac{\partial^{2} T^{*}}{\partial y^{*^{2}}}
$$

By introducing the non-dimensional variables

$$
\begin{aligned}
& y=\frac{u_{0} y^{*}}{v_{f}}, t=\frac{u_{0}^{2} t^{*}}{v_{f}}, u=\frac{u^{*}}{u_{0}}, \\
& \theta=\frac{T^{*}-T_{\infty}^{*}}{T_{w}^{*}-T_{\infty}^{*}}, C=\frac{C^{*}-C_{\infty}^{*}}{C_{w}^{*}-C_{\infty}^{*}}
\end{aligned}
$$

Eq.s (1), (3) and (10) reduces to the form

$$
\begin{aligned}
& \frac{\partial u}{\partial t}=a_{6} \frac{\partial^{2} u}{\partial y^{2}}+a_{7} \frac{\partial^{3} u}{\partial t \partial y^{2}}+G r a_{2} \theta-M^{2} a_{3} u \\
& +\mathrm{Gca}_{5} C-\frac{1}{K} u \\
& \frac{\partial \theta}{\partial t}=a_{4} \frac{\partial^{2} \theta}{\partial y^{2}} \\
& \frac{\partial C}{\partial t}=\frac{1}{S c} \frac{\partial^{2} C}{\partial y^{2}}+S o \frac{\partial^{2} \theta}{\partial y^{2}}
\end{aligned}
$$

Where

$$
\begin{aligned}
& x_{1}=\left[(1-\phi)+\phi\left(\frac{\rho_{s}}{\rho_{f}}\right)\right], x_{2}=\left[(1-\phi)+\phi\left(\frac{(\rho \beta)_{s}}{(\rho \beta)_{f}}\right)\right], \\
& x_{3}=\left[(1-\phi)+\phi\left(\frac{\left(\rho C_{p}\right)_{s}}{\left(\rho C_{p}\right)_{f}}\right)\right], \\
& x_{4}=\left[\frac{k_{s}+2 k_{f}-2 \phi\left(k_{f}-k_{s}\right)}{k_{s}+2 k_{f}+\phi\left(k_{f}-k_{s}\right)}\right], \\
& x_{5}=\left[1+\frac{3(\sigma-1) \phi}{(\sigma+2)-(\sigma-1) \phi}\right], \quad x_{6}=\frac{x_{4}}{x_{3}}, \\
& x_{7}=\left[(1-\phi)+\phi\left(\frac{\left(\rho \beta^{*}\right)_{s}}{\left(\rho \beta^{*}\right)_{f}}\right)\right], a_{1}=\frac{1}{(1-\phi)^{2.5} x_{1}}, \\
& a_{2}=\frac{x_{2}}{x_{1}}, a_{3}=\frac{x_{5}}{x_{1}}, a_{4}=\frac{1}{x_{3} \operatorname{Pr}}\left(x_{4}+N r\right), \\
& a_{5}=\frac{x_{7}}{x_{1}}, a_{6}=\frac{a_{1}}{1+\lambda_{1}}, a_{7}=\frac{\lambda_{2}}{1+\lambda_{1}}
\end{aligned}
$$

The corresponding initial and boundary conditions are

$$
\begin{aligned}
& t=0: u=0, \theta=0, C=0 \text { for all } y \geq 0, \\
& t>0: u=\lambda, \theta=1, C=1 \text { at } \mathrm{y}=0, \\
& t>0: u \rightarrow 0, \theta \rightarrow 0, C \rightarrow 0 \text { as } y \rightarrow \infty
\end{aligned}
$$




\section{METHOD OF SOLUTION}

Equations (12)-(14) are linear partial differential equations and are to be solved with the initial and boundary conditions (15). For this set of equations the exact solution is not possible and hence we solve these equations by finite-difference method. The equivalent finite difference schemes of equations for (12)-(14) are as follows:

$$
\begin{aligned}
& \frac{u_{i, j+1}-u_{i, j}}{\Delta t}=a_{6} \frac{u_{i-1, j}-2 u_{i, j}+u_{i+1, j}}{(\Delta y)^{2}} \\
& +a_{7} \frac{u_{i-1, j+1}-2 u_{i, j+1}+u_{i+1, j+1}-u_{i-1, j}+2 u_{i, j}-u_{i+1, j}}{\Delta t(\Delta y)^{2}} \\
& +G r a_{2} \theta_{i, j}-M^{2} a_{3} u_{i, j}+G c a_{5} C_{i, j}-\frac{1}{K} u_{i, j} \\
& \frac{\theta_{i, j+1}-\theta_{i, j}}{\Delta t}=a_{4} \frac{\theta_{i-1, j}-2 \theta_{i, j}+\theta_{i+1, j}}{(\Delta y)^{2}} \\
& \frac{C_{i, j+1}-C_{i, j}}{\Delta t}=\frac{1}{S c} \frac{C_{i-1, j}-2 C_{i, j}+C_{i+1, j}}{(\Delta y)^{2}} \\
& + \text { So } \frac{\theta_{i-1, j}-2 \theta_{i, j}+\theta_{i+1, j}}{(\Delta y)^{2}}
\end{aligned}
$$

Here, the suffix i refer to $y$ and $\mathrm{j}$ to time. The mesh system is divided by taking $\Delta y=0.1$. From the initial condition in $(15)$, we have the following equivalent: $u(i, 0)=0, \theta(i, 0)=0, C(i, 0)=0$ for all $\mathrm{i}$

The boundary conditions from (15) are expressed in finitedifference form as follows

$u(0, j)=\lambda, \theta(0, j)=1, C(0, j)=1$ for all $\mathrm{j}$

$u\left(i_{\max }, j\right)=0, \theta\left(i_{\max }, j\right)=0, C\left(i_{\max }, j\right)=0$ for all $\mathrm{j}(20)$

(Here $i_{\max }$ was taken as 200)

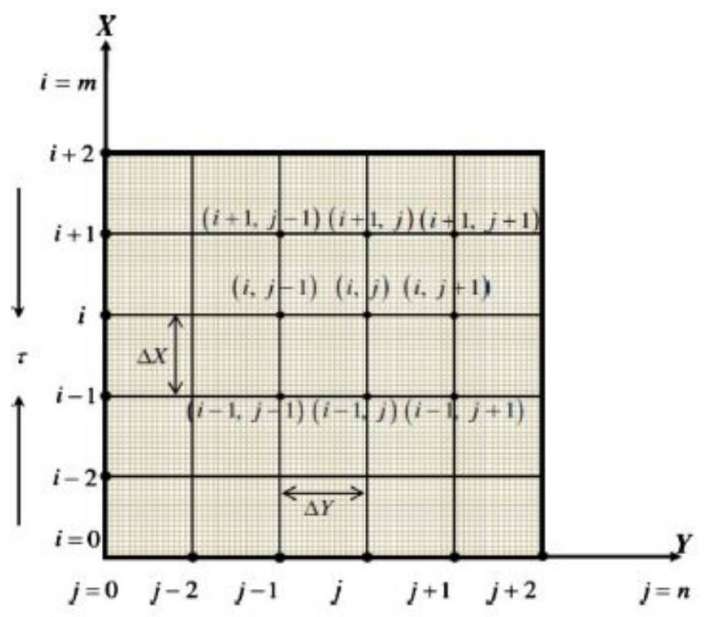

Fig. 2. Finite difference space grid

The velocity at the end of time step viz, $u(i, j+1)(i=1,200)$ is computed from (9) in terms of velocity, temperature and concentration at points on the earlier time-step. After that $\theta$ $(\mathrm{i}, \mathrm{j}+1)$ is computed from $(10)$ and then $\mathrm{C}(\mathrm{i}, \mathrm{j}+1)$ is computed from (11). The procedure is repeated until $\mathrm{t}=0.5$ (i.e. $\mathrm{j}=500$ ). During computation $\Delta \mathrm{t}$ was chosen as 0.001 .

\section{RESULTS AND DISCUSSION}

The characteristics of Jeffery nanofluid flow past a moving plate are pointed out with graphical representations.

\section{Validation of the results}

To validate the present methodology comparisons with previous studies has performed. Figure 3 shows the comparison of present results with that of Das and Jana (2015). A good coincidence is noticed in the absence of new parameters.

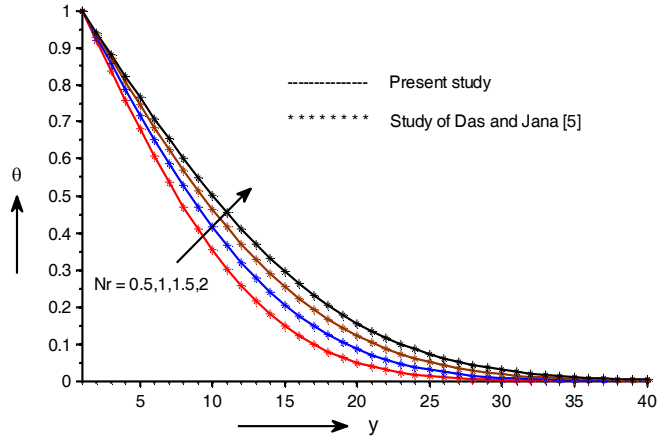

Fig. 3. Comparison of present results with previous literature without the effects of Jeffery parameter, porosity parameter, solutal Grashof number, Schmidt number and thermal diffusion.

Figure 4 presents the effect of Jeffery parameter on velocity distribution. It is noticed that the velocity increases with the increasing values of Jeffery parameter for the case of stationary plate $(\lambda=0)$ and back-moving plate $(\lambda=-1)$ but a reverse nature is noticed in the case of forth-moving plate $(\lambda=1)$. Figures 5,6 depict the effects of Grashof number of heat transfer and mass transfer respectively on velocity distribution. Grashof number represents the effect of free convection currents. It is noticed that the velocity increases with increasing values of both the numbers for the cases of stationary plate $(\lambda=0)$ as well as moving plate $(\lambda= \pm 1)$. This behavior is due to the favorable pressure gradient which accelerates the fluid in the boundary layer. The impact of radiation parameter on the velocity is demonstrated in Figure 7. The velocity of the fluid enhances as the value of radiation parameter increases for both the cases of stationary plate $(\lambda=0)$ and moving plate $(\lambda= \pm 1)$. Thermal radiation provides a supporting force which accelerates the fluid particles at the locality of the plate and hence the rising of velocity. It is obvious from figure 8 that the flow velocity comes down with increasing values of Prandtl number. The reason behind this nature is that the fluid of low Prandtl number has high thermal diffusivity and hence attains higher temperature in steady 
state, which in turn means more buoyancy force. Figure 9 reveals that the fluid velocity accelerates under the impact of magnetic parameter. This trend is due to fact that the transverse magnetic field sets in Lorentz force, which retards the fluid velocity. Figure 10 depicts the influence of Schmidt number on fluid velocity. The Schmidt number embodies the ratio of the momentum diffusivity to the species (mass) diffusivity. It physically relates to the comparative thickness of the hydrodynamic boundary layer and mass-transfer boundary layer. It is observed that the velocity field decreases when Schmidt number increases for the cases of stationary plate $(\lambda=0)$ as well as moving plate $(\lambda= \pm 1)$. The influence of Soret number on velocity field is demonstrated in figure 11. It is clear that the velocity grows under the existence of thermal diffusion for all the three cases. Figure 12 shows the effect of time on velocity distribution. It is observed that the velocity grows as time elapses. The velocity enhances as the values of porosity parameter increases for the case of stationary plate $(\lambda=0)$ and forth-moving plate $(\lambda=1)$ but a reverse nature is noticed in the case of back-moving plate $(\lambda=-1)$ which is evident from figure 13. Figure 14 shows the effect of solid volume fraction on velocity distribution. The velocity decreases by increasing the volume of nano particles for both the cases of stationary plate $(\lambda=0)$ and moving plate $(\lambda= \pm 1)$.

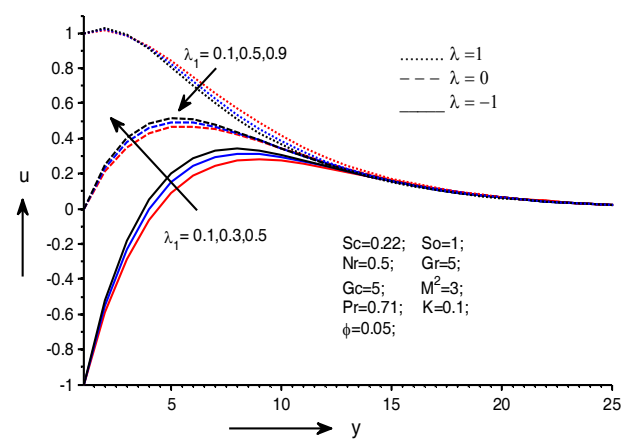

Fig. 4. Effect of Jeffery parameter on velocity

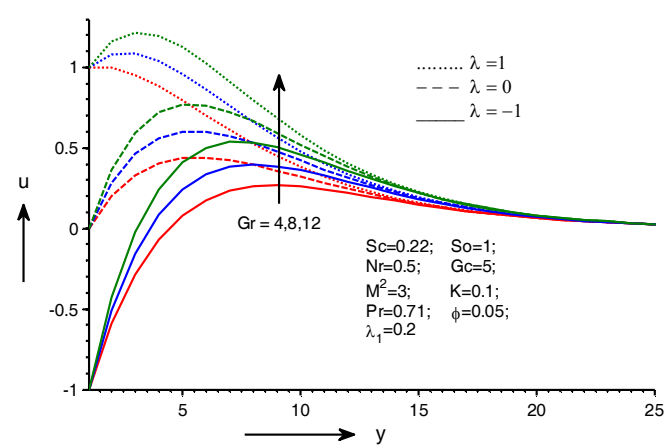

Fig.5. Effect of Grashof number on velocity

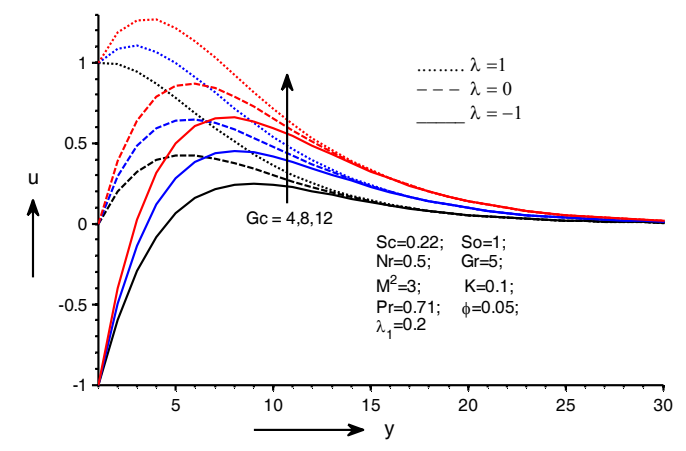

Fig. 6. Effect of modified Grashof number on velocity

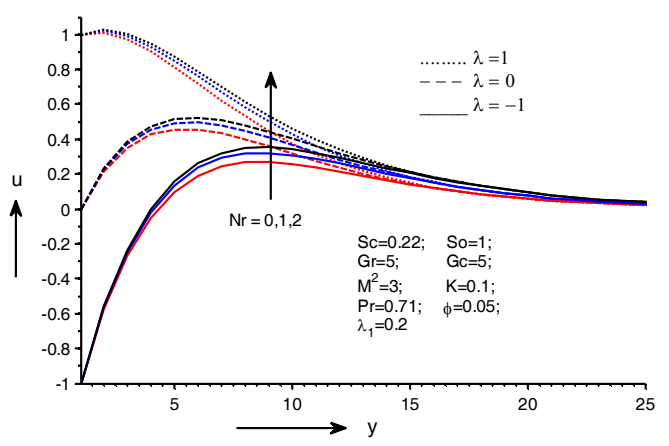

Fig. 7. Effect of radiation parameter on velocity

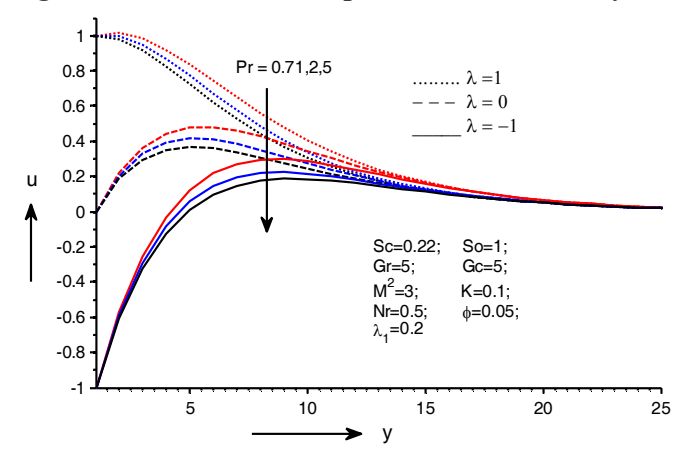

Fig. 8. Effect of Prandtl number on velocity

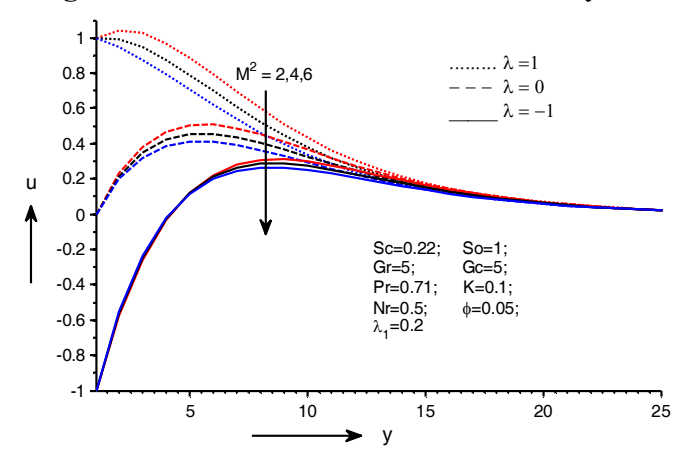

Fig. 9. Effect of magnetic parameter on velocity 


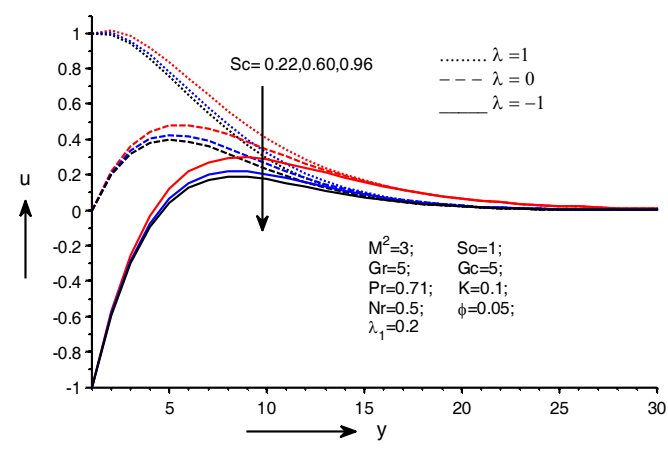

Fig.10. Effect of Schmidt number on velocity

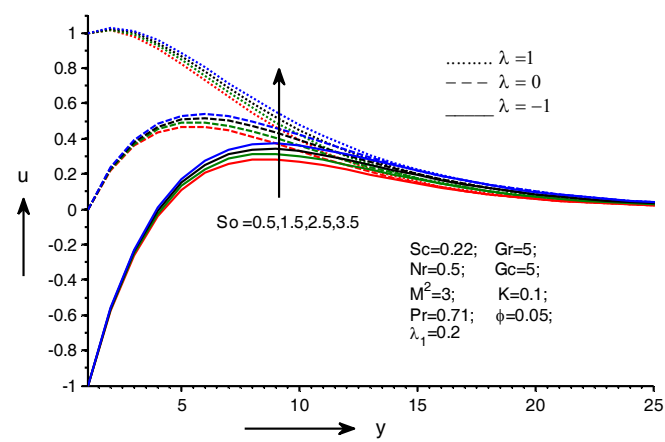

Fig. 11. Effect of Soret number on velocity

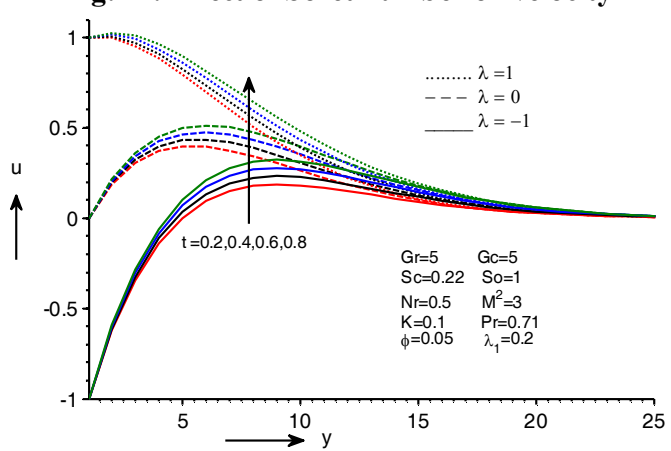

Fig. 12. Effect of time on velocity

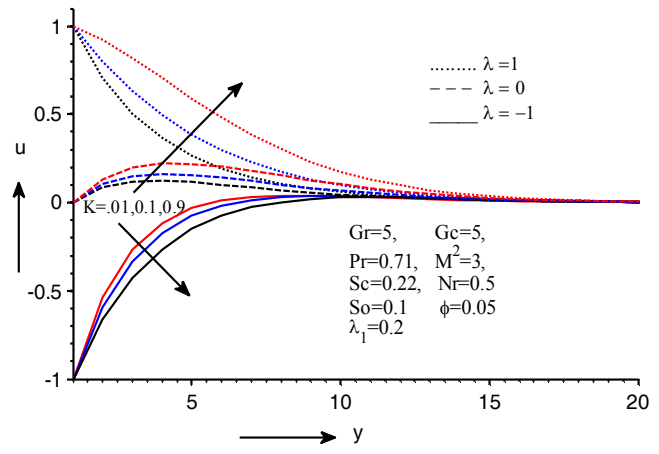

Fig. 13. Effect of Porosity parameter on velocity

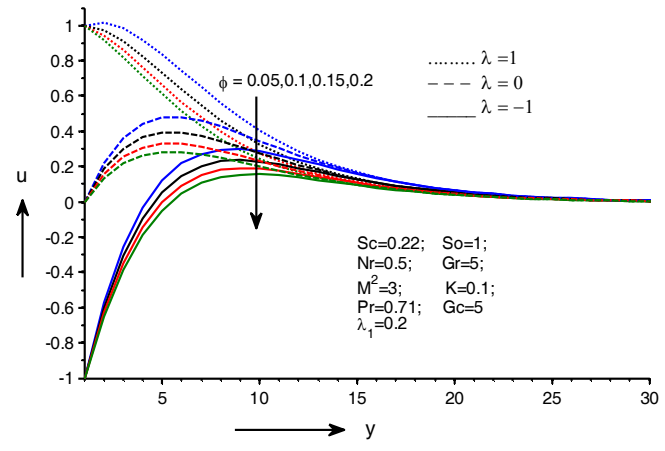

Fig. 14. Effect of solid volume fraction on velocity

The fluid temperature rises under the existence of thermal radiation which is evident from figure 15. This behavior is due to the fact that an increase in radiation parameter corresponds to the release of heat energy from the flow region. It is clear from figure 16 that the fluid temperature rises with enlarging values of volume fraction parameter. The thermal boundary layer for $\mathrm{Cu}$-water nanofluid is higher than for pure water $(\phi=0)$ since copper has more thermal conductivity and its addition to the water based fluid increases the thermal conductivity for the fluid. Consequently the thickness of the thermal boundary layer enhances. This trend coincides with the physical nature of nanoparticles. This observation shows the significance of nanofluids in the cooling and heating processes. The effect of time on variation of fluid temperature is shown in figure 17. It is observed that the fluid temperature rises as time elapses. The effect of Prandtl number on the variation of fluid temperature is exhibited in figure 18. It is noticed that the fluid temperature falls down as the Prandtl number enlarges. It is obvious that a higher Prandtl number fluid has relatively low thermal conductivity which causes a reduction in the conduction and there by the thermal boundary layer thickness. As a result the fluid temperature decreases.

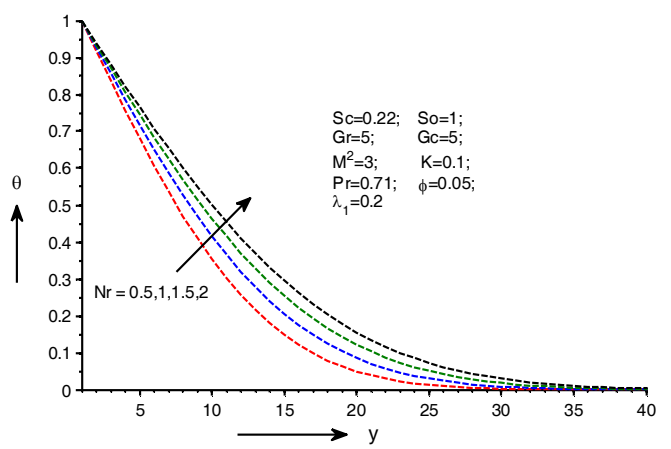

Fig. 15. Effect of radiation parameter on temperature 


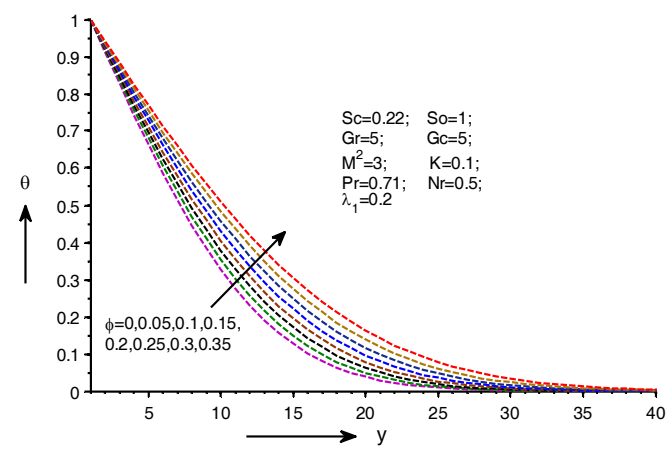

Fig.16. Effect of Solid volume parameter on temperature

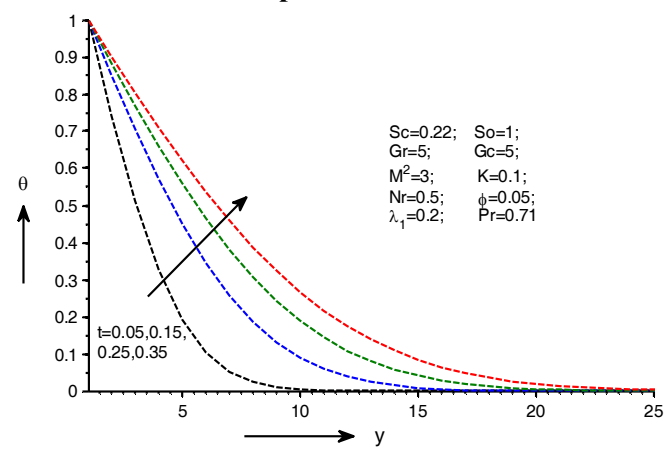

Fig. 17. Effect of time on temperature

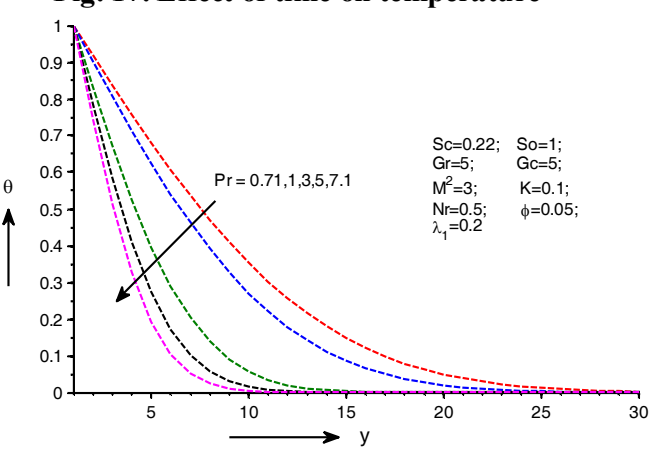

Fig. 18. Effect of Prandtl number on temperature

Figures 19 and 20 reveal the impact of Schmidt number and Soret number on the species concentration. It can be seen that the concentration field become thinner under the effect of Schmidt number, but a reverse trend is noticed in the case of Soret effect. Figure 21 shows the variation of the concentration distribution under the influence of time. It is observed that the fluid concentration increases as the time increases. The fluid concentration becomes thinner with increasing values of radiation parameter. This is clearly shown in figure 22 . Figure 23 reveals the variation of the concentration distribution under the influence of volume fraction parameter. It is observed that the fluid concentration decreases as the solid volume fraction increases.

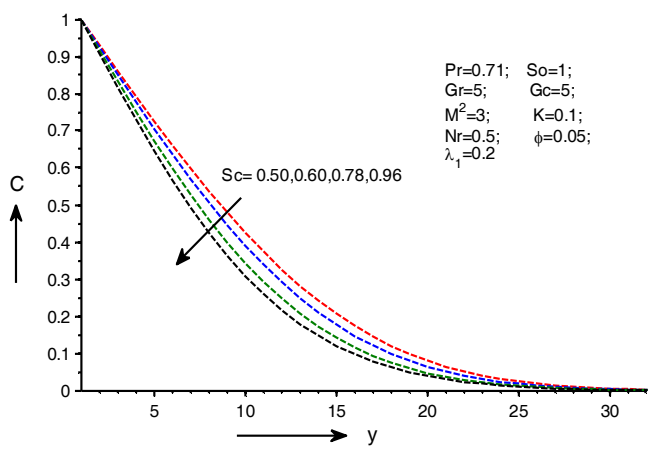

Fig. 19. Effect of Schmidt number on concentration

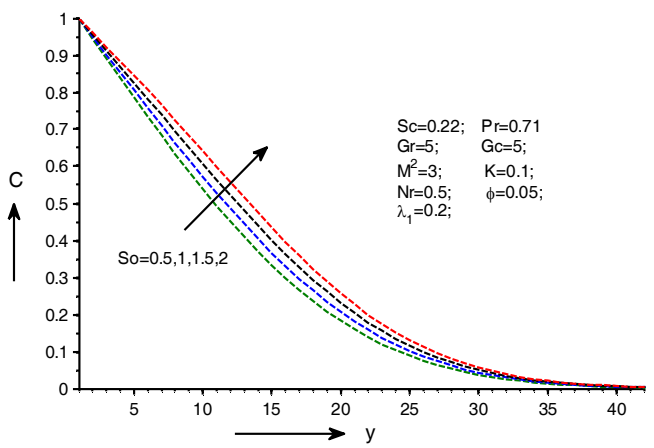

Fig. 20. Effect of soret number on concentration

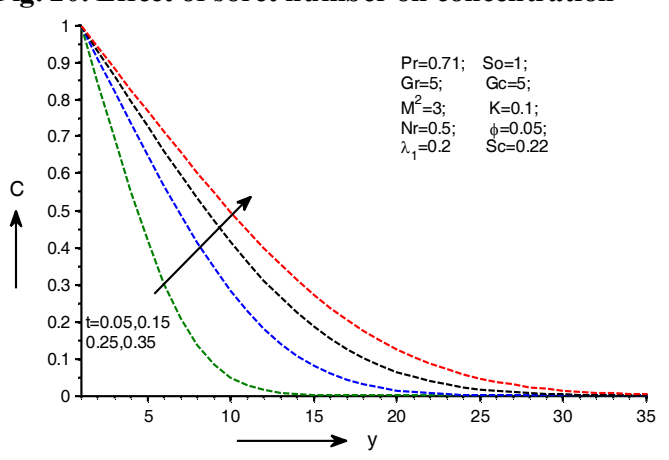

Fig. 21. Effect of time on concentration

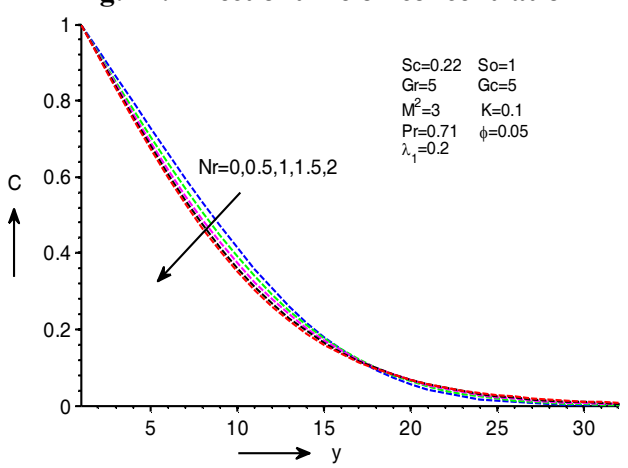

Fig. 22. Effect of radiation parameter on concentration 


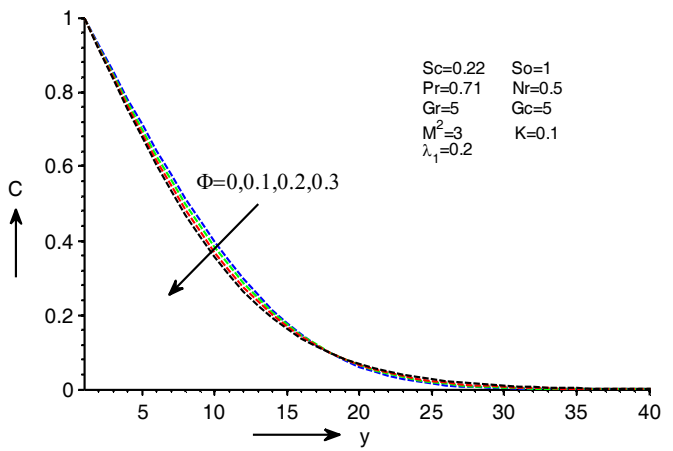

Fig. 23. Effect of Solid volume fraction on concentration

Researchers are very much interested to evaluate the shear stress (or skin friction) for the sake of engineering purposes. The non-dimensional shear stress at the plate $y=$ 0 due to the flow is given by the relation

$$
\tau=-\left(\frac{d u}{d y}\right)_{y=0}
$$

Numerical results of the non-dimensional skin friction are presented in figures 24-29 under the effects of Jeffery parameter $\lambda_{1}$, Grashof number Gr, modified Grashof number Gc, Schmidt number Sc and Soret number So. The effect of Jeffery parameter of heat and mass transfer on skin friction is shown in figure 24. It is observed that the skin friction decreases with the increasing values of Jeffery parameter $\lambda_{1}$ for the case of stationary plate $(\lambda=0)$ and back-moving plate $(\lambda=-1)$ but a reverse nature is noticed in the case of forth-moving plate $(\lambda=1)$.The effects of the Grashof numbers of heat and mass transfer on skin friction are displayed in figures 25-26. It can be seen that the skin friction reduces for increasing values of both the numbers for the stationary plate $(\lambda=0)$ and also moving plate $(\lambda=$ \pm 1 ). This is because the positive buoyancy force acts like a favorable pressure gradient and hence the fluid in the boundary layer gets accelerated. As a consequence the hot fluid near the plate surface passes away faster as Grashof number Gr increases and so the shear stress at the plate reduces. This result coincides with the results of Das and Jana [5] .The variation of shear stress with the effect of Schmidt number can be seen in figure 27. An increase in Schmidt number leads to an increase in skin friction for the stationary plate $(\lambda=0)$ as well as moving plate $(\lambda= \pm 1)$. Figures 28 illustrate the behavior of shear stress at the plate which states that the rising values of Soret number results in reduction of shear stress in both the cases.

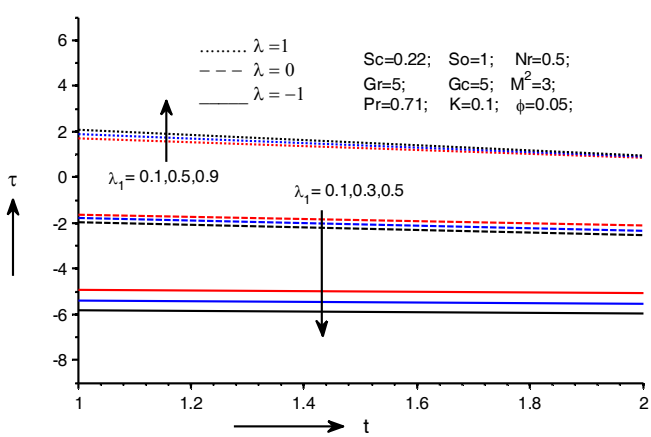

Fig. 24. Effect of Jeffery parameter on skin friction

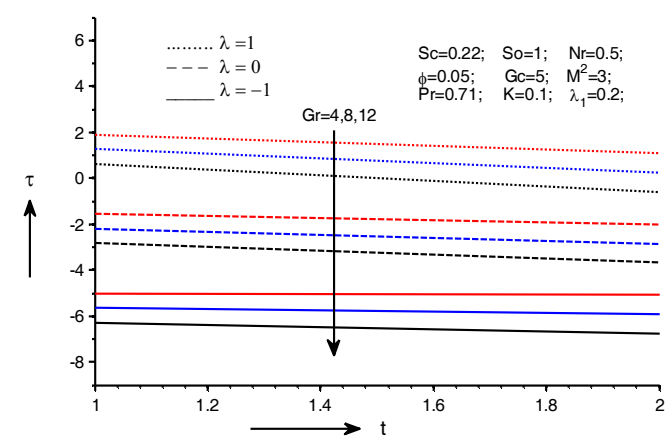

Fig. 25. Effect of Grashof number on skin friction

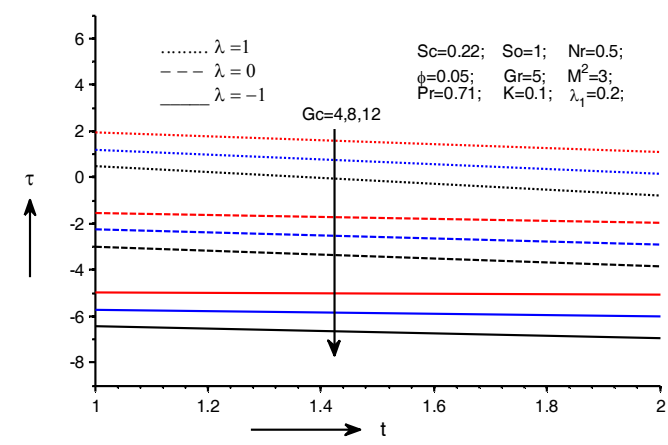

Fig. 26. Effect of modified Grashof number on skin friction

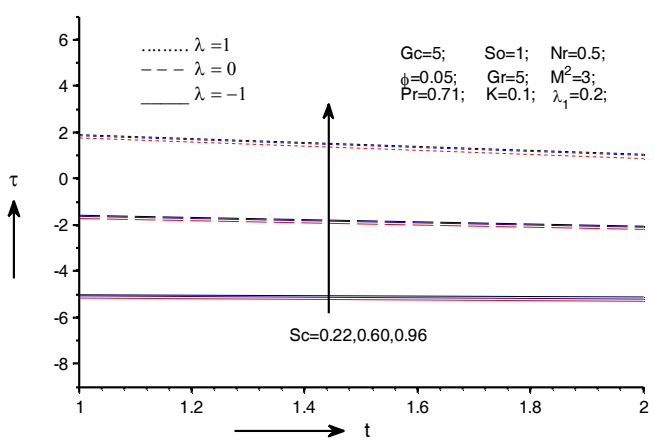

Fig. 27. Effect of Schmidt number on skin friction 


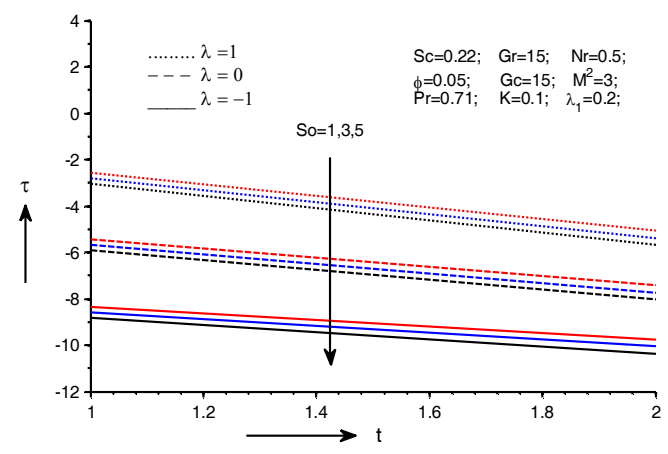

Fig. 28. Effect of Soret number on skin friction

The dimensionless rate of heat transfer in terms of Nusselt number is given by

$N u=-\left(\frac{d \theta}{d y}\right)_{y=0}$

Numerical values of non-dimensional rate of heat transfer at the plate $y=0$ are displayed in figures 29-31 for different values of radiation parameter Nr, Prandtl number Pr and Solid volume fraction parameter $\phi$. From figure 29 it is evident that the rate of heat transfer decreases with the increasing values of radiation parameter. This happens due to the fact that as thermal radiation increases, the dominance effect of temperature gradient increases. Consequently, the rate of heat transfer increases. As the Prandtl number increases the rate of heat transfer enhances. It is evident from figure 30. Figure 31 illustrates that the rate of heat transfer decreases as volume fraction parameter enlarges. This can be realized with the increase in thermal conductivity under the effect of solid volume fraction of nanoparticles. On the other hand, the thermal boundary layer thickness decreases with increase of nanoparticle volume fraction and in turn the rate of heat transfer increases with increase of solid volume fraction parameter.

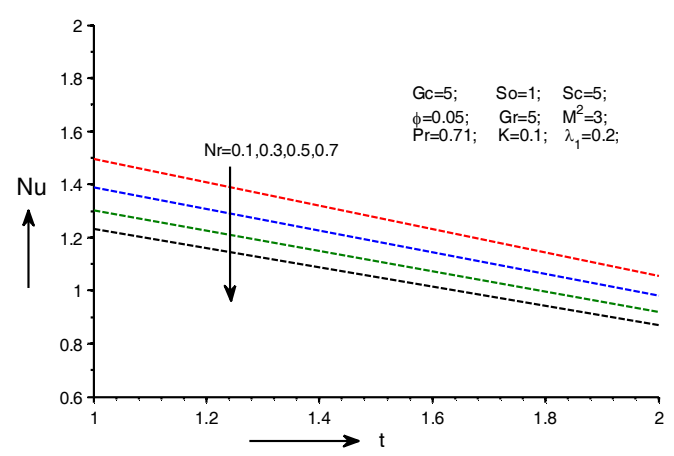

Fig. 29. Effect of radiation parameter on Nusselt number

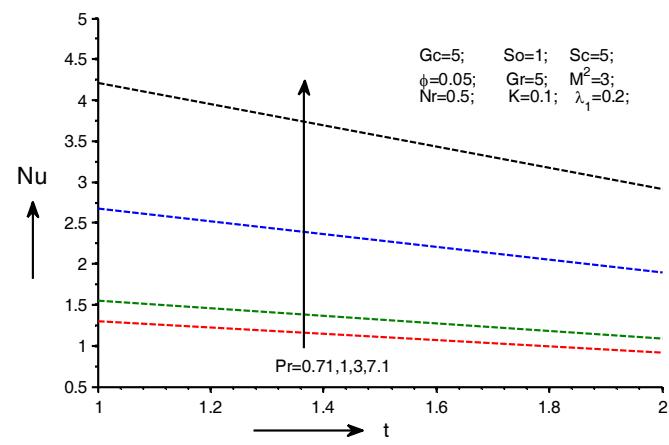

Fig. 30. Effect of Prandtl number on Nusselt number

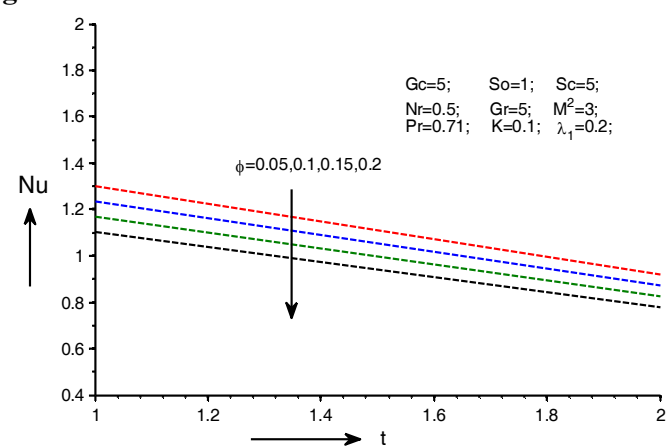

Fig. 31. Effect of Solid volume fraction on Nusselt number

The dimensionless rate of mass transfer in terms of Sherwood number is given by $S h=-\left(\frac{d C}{d y}\right)_{y=0}$

The effects on the non-dimensional rate of mass transfer at the plate $\mathrm{y}=0$ are shown in figures $32-35$ for several values of Soret number So, Schmidt number Sc, radiation parameter $\mathrm{Nr}$, and volume fraction parameter $\phi$. Figures 32 $\& 33$ depict the variation of the rate of mass transfer with the effect of Soret number and Schmidt number. The Sherwood number decreases with the rising values of Soret number. But a reverse trend is noticed in the case of Schmidt number. Figures $34 \& 35$ reveal the variation of Sherwood number under the influence of volume fraction parameter and radiation parameter. It is clear that the rate of mass transfer increases with increasing values of both the parameters. 


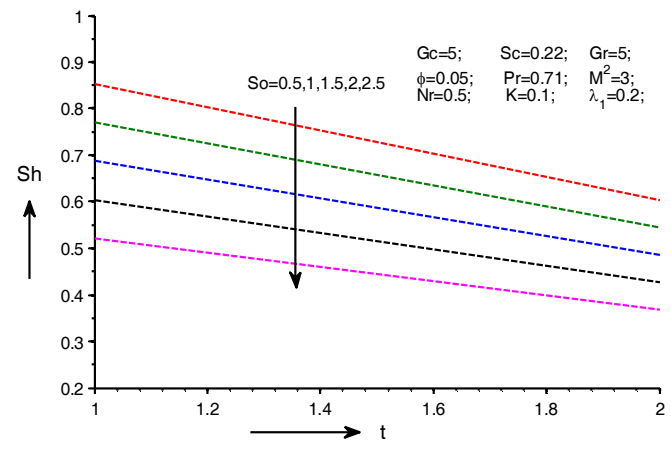

Fig. 32. Effect of Soret number on Sherwood number

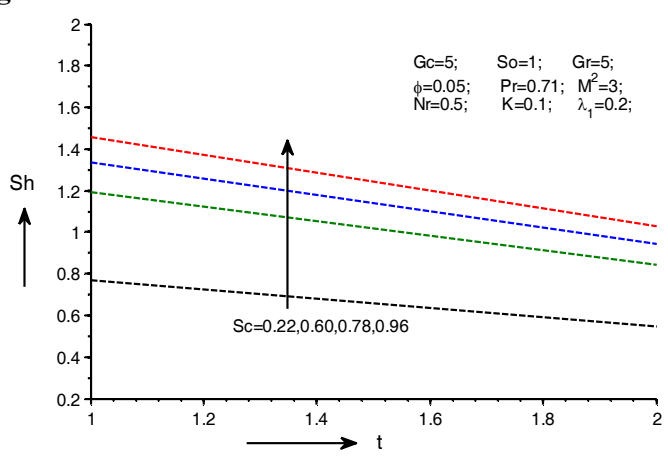

Fig. 33. Effect of Schmidt number on Sherwood number

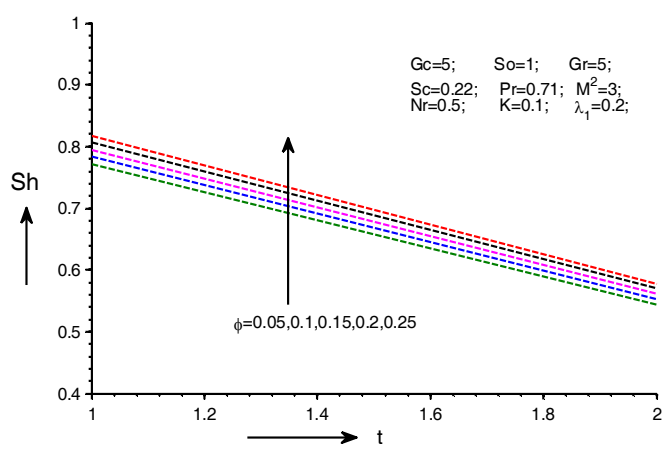

Fig. 34. Effect of Solid volume fraction on Sherwood number

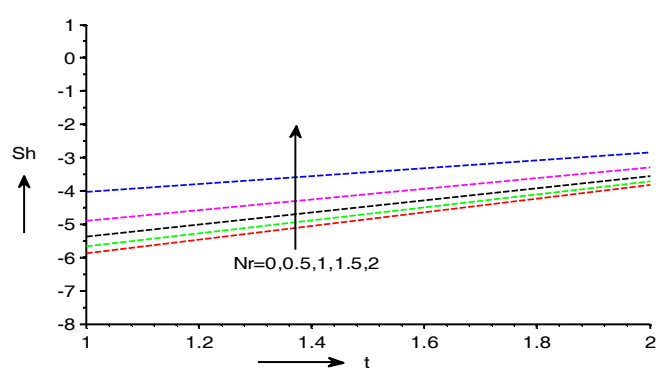

Fig. 35. Effect of radiation parameter on Sherwood number

\section{CONCLUSIONS}

The most interesting points can be summarized as follows:

- The fluid velocity increases under the influence of Jeffery parameter for the case of stationary plate and back-moving plate, but a reverse nature is notified in the case of forth-moving plate.

- Velocity increases with improved values of radiation parameter and Soret number whereas opposite movement is revealed in the case of Prandtl number and Schmidt number.

- The velocity enhances as the values of porosity parameter increases for the case of stationary plate and forth-moving plate but a reverse nature is noticed in the case of back-moving plate.

- An increase in radiation parameter and solid volume fraction leads to enhance the fluid temperature.

- $\quad$ Rising values of Soret number serves to improve the species concentration.

- Increasing values of Schmidt number enhanced the shear stress at the plate but a reverse trend is noticed in the case of Soret number.

- The rate of heat transfer enlarged under the impact of radiation parameter and volume fraction.

- Sherwood number increases for rising values of Soret number and falling values of Schmidt number.

\section{REFERENCES}

Abbasi, F. M., S. A. Shehzad, T. Hayat, A. Alsaedi, and M. A. Obid (2015). Influence of heat and mass flux conditions in hydromagnetic flow of Jeffrey nanofluid. AIP Adv. 5, 1-12.

Al- Khafajy, D. G. S (2016). Effects of heat transfer on MHD oscillatory flow of Jeffrey Fluid with variable viscosity through porous medium. Advances in Applied Science Research 7(3), 179-186.

Chandra Reddy, P., M. C. Raju, G. S. S. Raju (2015). Thermal and solutal buoyancy effect on MHD boundary layer flow of a visco-elastic fluid past a porous plate with varying suction and heat source in the presence of thermal diffusion. Journal of Applied \& Computational Mathematics 4(5), 1-7.

Choi, S. U. S. (1995). Enhancing thermal conductivity of fluids with nanoparticles developments and applications of non-Newtonian flows. ASME FED 231/MD 66, 99-105.

Choi, S. U. S., Z. G. Zhang, W. Yu, F. E. Lockwood and E. A. Grulke (2001). Anomalously thermal conductivity enhancement in nanotube suspensions. App. Phy. Lett. $79,2252-2254$. 
Dalir, N., M. Dehsara, and S. S. Nourazar (2015). Entropy analysis for magnetohydrodynamic flow and heat transfer of a Jeffrey nanofluid over a stretching sheet. Energy 79, 351-362.

Das S. K. and S. U. Choi (2009). A review of heat transfer in nanofluids. Adv. Heat Transfer 46, 119.

Das, S. and R. N. Jana (2015). Natural convective magnetonanofluid flow and radiative heat transfer past a moving vertical plate. Alexandria Engg. Jou. 54, 5564.

Eastman, J.A. S. U. S. Choi, S. Li, W. Yu, L. J. Thompson (2001). Anomalously increased effective thermal conductivities of ethylene glycol-based nanofluids containing copper nanoparticles. App. Phy. Lett. 78, $718-720$.

Feroz Ahmed Soomro, Rizwan-ul-Haq, Z. H. Khan, Qiang Zhang (2017a). Numerical study of entropy generation in MHD water based carbon nanotubes along an inclined permeable surface. Eur. Phys. J. Plus 132 (412).

Feroz Ahmed Soomro, Rizwan -ul-Haq, Zafar Hayat Khan, Qiang Zhang (2017b). Passive control of nanoparticles due to convective heat transfer of Prandtl fluid model at the stretching surface. Chin. J. Phys. 55, 15611568 .

Ganesh Kumar, K., N. G. Rudraswamy, B. J. Gireesha, Rizwan -ul-Haq (2017). Effects of mass transfer on MHD three dimensional flow of a Prandtl liquid over a flat plate in the presence of chemical reaction. Results Phys. 7, 3465-3471.

Hashim, Masood Khan, Ali Saleh Alshomrani, Riwan U1 Haq (2017). Investigation of dual solutions in flow of a non-Newtonian fluid with homogeneous heterogeneous reactions: critical points. Eur. J. Mech./B Fluids 68, 30-38.

Hayat, T., T. Muhammad, S. A. Shehzad, and A. Alsaedi (2015). A mathematical study for three-dimensional boundary layer flow of Jeffrey nanofluid. Zeitschrift für Naturforsch. A. 70(4), 225-233.

Hayat, T., S. Asad, M. Qasim and A. A. Hendi (2012). Boundary layer flow of a Jeffrey fluid with convective boundary conditions. Int J Numer Methods Fluids 69, $1350-2$.

Hayat, T., T. Muhammad, S. A. Shehzad, and A. Alsaedi, (2016).Three-dimensional flow of Jeffrey nanofluid with a new mass flux condition. J. Aerosp. Eng 29(2), 1-8

Kakac S. and A. Pramuanjaroenkij (2009). Int Review of convective heat transfer enhancement with nanofluids. Jou. Heat Mass Transfer 52, 3187-3196.
Kavita, K., Ramakrishna Prasad, K., Aruna Kumari B (2012). Influence of heat transfer on MHD oscillatory flow of Jeffrey Fluid in a channel. Advances in Applied Science Research 3(4), 2312-2325.

Khan, I. (2015) . A note on exact solutions for the unsteady free convection flow of a Jeffrey fluid. Z. Naturforsch 70(6), 272-284.

Mabood, F., R. Abdel-Rahman, G. Lorenzini (2016). Numerical study of unsteady Jeffrey fluid flow with magnetic field effect and variable fluid properties. Journal of Thermal Science and Engineering Applications 8, 1-9.

Maqbool K. , A. B. Mann, M. H. Tiwana (2017). Unsteady MHD convective flow of a Jeffery fluid embedded in a porous medium with ramped wall velocity and temperature. Alexandria Engineering Journal .

Mehmood , R., S. Nadeem, S. Saleem and N. S. Akbar (2017). Flow and heat transfer analysis of Jeffery nano fluid impinging obliquely over a stretched plate. Journal of the Taiwan Institute of Chemical Engineers $74,49-58$.

Ramzan, M., M. Bilal and Jae Dong Chung (2017). Effects of thermal and solutal stratification on Jeffrey magneto-nanofluid along an inclined stretching cylinder with thermal radiation and heat generation/absorption. International Journal of Mechanical Sciences 131, 317-324.

Rashidi, M. M., S. Reddy and K. Naikotib (2017). MHD flow and heat transfer characteristics of Williamson nanofluid over a stretching sheet with variable thickness and variable thermal conductivity, Transactions of A. Razmadze Mathematical Institute $171,195-211$

Rohit Sharma, S. M. Hussain, Hitesh Joshi and G. S. Seth (2017). Analysis of radiative magneto-nanofluid over an accelerated plate in a rotating medium with Hall effects. Diffusion Foundations 11, 129-145.

Seth, G. S. and M. K. Mishra (2017). Analysis of transient flow of MHD nanofluid past a nonlinear stretching sheet considering Navier's slip boundary condition. Advanced Powder Technology 28, 375-384.

Seth, G. S., B. Kumbhakar and R. Sharma (2016). Unsteady MHD free convection flow with Hall effects of a radiating and heat absorbing fluid past a moving vertical plate with variable ramped temperature. $J$. Egypt. Maths. Soc. (JOEMS) 24(3), 471-478.

Seth, G. S., R. Sharma, B. Kumbhakar and R. Tripathi (2017a). MHD stagnation point flow over exponentially stretching sheet with exponentially moving free-stream, viscous dissipation, thermal 
radiation and non-uniform heat source/sink. Diffusion Foundations 11, 182-190.

Seth, G. S., A. Bhattacharyya and R. Tripathi (2017b). Effect of Hall current on MHD natural convection heat and mass transfer flow of rotating fluid past a vertical plate with ramped wall temperature. Frontiers in Heat and Mass Transfer (FHMT) 9, 21 (12 pages) .

Shehzad, S. A., T. Hayat, A. Alsaedi, and M. A. Obid (2014). Nonlinear thermal radiation in threedimensional flow of Jeffrey nanofluid: A Model for Solar Energy, Appl. Math. Comput 248, 273-286.

Shehzad, S. A., T. Hayat, and A. Alsaedi (2015). MHD flow of Jeffrey nanofluid with convective boundary conditions. J. Brazilian Soc. Mech. Sci. Eng 37(3), 873-883.

Shehzad, S. A., Z. Abdullah, A. Alsaedi, F.M. Abbasi, and T. Hayat (2016). Thermally radiative threedimensional flow of Jeffrey nanofluid with internal heat generation and magnetic field. J. Magn. Magn. Mater 397, 108-114.
Sheikholeslami, M., T. Hayat . and A. Alsaedi (2016). Numerical study for external magnetic source influence on water based nanofluid convective heat transfer. Int. J. Heat Mass Transf.

Sheikholeslami, M., D. D. Ganji (2017). Transportation of MHD nanofluid free convection in a porous semi annulus using numerical approach. Chem. Phys. Lett $669,202-210$.

Singh, J. K., G. S. Seth and S. G. Begum (2017). Unsteady MHD natural convective flow of a rotating fluid over an infinite vertical plate due to oscillatory movement of the free-stream with hall and ion-slip currents. Diffusion Foundations 11, 146-161.

Sreenadh, S., M. Krishnamurthy, E. Sudhakara, G. Gpoi Krishna and D. Venkateswarlu Naidu (2015). MHD free surface flow of a Jeffery fluid over a deformable porous layer. Global Journal of Pure and Applied Mathematics 11, 3889-3903.

Syakila A. and I. Pop (2010). Mixed convection boundary layer flow from a vertical flat plate embedded in a porous medium filled with nanofluids. Int Comm Heat Mass Transfer 37(9), 87-91. 